\title{
The GALEX View of Supernova Hosts
}

\author{
James D. Neill*, Mark Sullivan ${ }^{\dagger}$ and Mark Seibert** \\ ${ }^{*}$ California Institute of Technology, 1200 E. California Blvd., Pasadena, CA, 91214, USA \\ ${ }^{\dagger}$ University of Oxford, Denys Wilkinson Building, Keble Road, Oxford, OXI 3RH, UK \\ ${ }^{* *}$ The Observatories of the Carnegie Institute of Washington, 813 Santa Barbara Street, Pasadena, \\ CA, 91101, USA
}

\begin{abstract}
.
We exploit the accumulating, high-quality, multi-wavelength imaging data of nearby supernova (SN) hosts to explore the relationship between SN production and host galaxy evolution. The Galaxy Evolution Explorer (GALEX, [1]) provides ultraviolet (UV) imaging in two bands, complementing data in the optical and infra-red (IR). We compare host properties, derived from spectral energy distribution (SED) fitting, with nearby, well-observed SN Ia light curve properties. We also explore where the hosts of different types of SNe fall relative to the red and blue sequences on the galaxy UV-optical color-magnitude diagram (CMD, [2]). We conclude that further exploration and larger samples will provide useful results for constraining the progenitors of SNe.
\end{abstract}

Keywords: galaxies: evolution - supernovae: general

PACS: $97.60 . \mathrm{Bw}$

\section{INTRODUCTION}

The diagnostic power of GALEX imaging for galaxy evolution is revealed in a plot of simple stellar population age versus wavelength shown at the top of Figure 1 in Martin et al. [1]. This plot covers the UV to the IR and demonstrates that the UV is most sensitive to star formation on time scales of $\sim 10^{8} \mathrm{yr}$. On the same figure, we see the flux of stellar populations with differing Scalo b $(\equiv \dot{M} /\langle\dot{M}\rangle$, where $\dot{M}$ is the star formation rate in $\mathrm{M}_{\odot} \mathrm{yr}^{-1}$ ) plotted against wavelength. The UV shows more discriminating power than other wavelengths. GALEX imaging is sensitive over at least five orders of magnitude of Scalo $b$, from $b=10$ down to $b=10^{-3}$. Another illustration of the leverage of UV imaging on galaxy evolution is shown in the galaxy CMD in Figure 9 of Wyder et al. [2]. Plotting galaxy near-UV minus r-band (NUV-r) color versus absolute r-band magnitude $\left(\mathrm{M}_{r}\right)$ clearly separates the blue and red sequences of galaxies, affording an exploration of the transition galaxies between these sequences. Cosmic downsizing of galaxies hosting active star formation is also illustrated using GALEX data, by plotting the surface density of star formation versus redshift (Figure 18 of Martin et al. [3]). These evolution diagnostics are further enhanced when comparing a large local galaxy sample [4] in the restframe UV with deep optical high-z galaxy surveys that observe the younger universe also in the restframe UV.

What do these diagnostics tell us about galaxies hosting various types of SNe? To begin to answer this, we analyze a preliminary set of $\sim 100$ galaxies hosting both core-collapse (CC) and thermonuclear (type Ia) SNe. We derive their integrated UV photometry using a preliminary version of the GALEX Large Galaxy Atlas (GLGA, [5]).

\footnotetext{
CP1111, Probing Stellar Populations Out to the Distant Universe, Cefali 2008, Proceedings of the International Conference edited by L. A. Antonelli, E. Brocato, M. Limongi, N. Menci, G. Raimondo, and A. Tornambè 1009 American Institute of Physics 978-0-7354-0648-3/09/\$25.00
} 


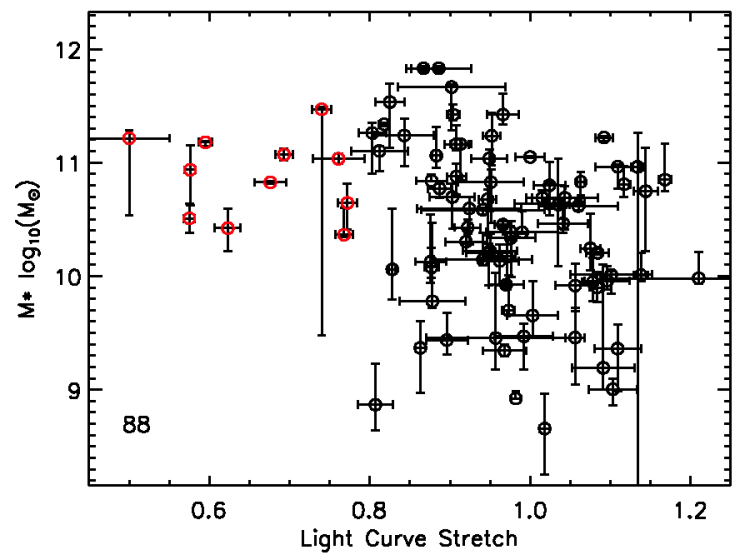

FIGURE 1. Derived host stellar mass versus SN Ia light curve stretch for the hosts of low-redshift, well-observed SNe Ia. Note that for hosts of SNe Ia with stretch $<0.8$, the mass range is much narrower than hosts of higher-stretch SNe Ia.

To this we add integrated optical and IR photometry extracted from online databases ${ }^{1}$. We fit the resulting SEDs with star formation history models $[6,7,8]$, and plot the photometry on diagnostic diagrams to explore the link between host evolution and SN production.

\section{HOST PHYSICAL PROPERTIES FROM SED FITS}

We first examine the SED fit results for a set of low-z galaxies hosting well-observed $\mathrm{SNe}$ Ia and plot the results versus the light-curve stretch [9], which parameterizes the evolution timescale of the SN luminosity. Figure 1 illustrates the trend in host stellar mass with stretch. It appears that low-stretch, and thus low-luminosity, SNe Ia prefer hosts in a narrow mass range of $10.2<\log \mathrm{M}_{*}<11.5$ (with $\mathrm{M}_{*}$ in $\mathrm{M}_{\odot}$ ), while the higher stretch SNe Ia are found in hosts over a much larger range: $8.5<\log \mathrm{M}_{*}<12$.

Figure 2 shows host specific star formation rate ( $\mathrm{SSFR} \equiv \mathrm{SFR} / \mathrm{M}_{*}$ in $\mathrm{yr}^{-1}$ ) versus stretch. We find that the hosts of low-luminosity SNe Ia have a range of sSFR indicating a range of host morphology as was found in other studies (e.g., [10]). However, here we see indications of a trend in SSFR for hosts of SNe with stretch $<0.8$. These trends in $\mathrm{M}_{*}$ and sSFR are also apparent in much simpler diagnostic plots such as absolute $\mathrm{K}$-band magnitude and far-UV minus K-band (FUV-K) color versus stretch, indicating that they are not artifacts of the SED-fitting process. The statistical significance of these trends will be quantified after the photometry and SED-fitting are further refined [11].

${ }^{1}$ http://nedww .ipac.caltech.edu/and http://irsa.ipac.caltech.edu/ 


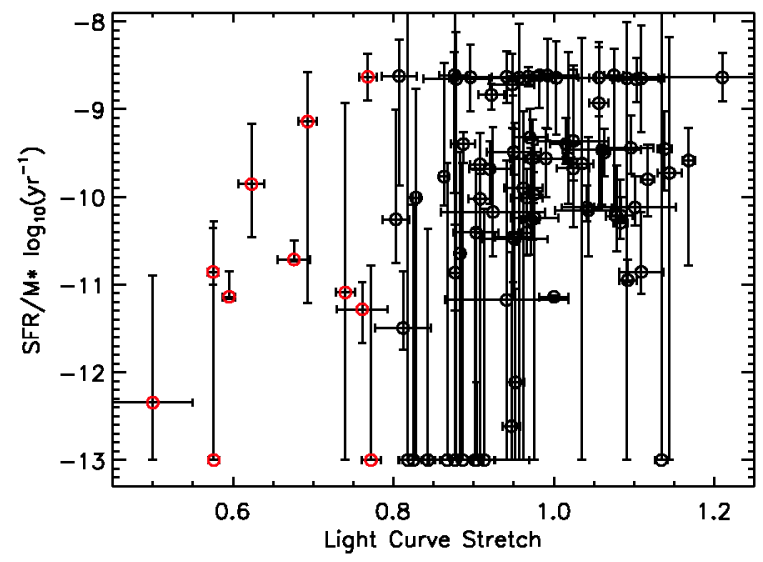

FIGURE 2. Derived host specific star formation rate $\left(\mathrm{SFR} / \mathrm{M}_{*}\right)$ versus stretch for the hosts of lowredshift, well-observed SNe Ia. For hosts of SNe Ia with $s<0.8$, there appears to be a downward trend in $\mathrm{SFR} / \mathrm{M}_{*}$ as stretch declines.

\section{THE GALAXY COLOR-MAGNITUDE DIAGRAM}

We next examine where local SN hosts fall on the galaxy NUV-r versus $\mathrm{M}_{r}$ plane as a function of SN type. Figure 3 plots SN hosts coded for SN type along with the red and blue galaxy sequences and should be compared with Figure 9 in Wyder et al. [2].

We see that all but one (SN2006ee) of the CC SNe (types II and Ibc) are found in blue sequence galaxies, while the SNe Ia appear in galaxies in both sequences and in between. In the blue sequence, the type II SN hosts dominate on the bluest side, while the type Ibc hosts seem to extend into the 'green valley' between the two sequences. Currently, the sample is too small to draw strong conclusions about the hosting populations of these $\mathrm{SNe}$, but the distribution in this diagram appears to contain information that will be useful in our efforts to constrain progenitors for various types of SNe. Once our sample is large enough, we can sub-divide the basic SN types to see if more structure in this diagram is revealed.

\section{SUMMARY}

We have seen that SED fitting and diagnostic plots derived from integrated photometry of SN host galaxies have the potential to tell us about the host evolutionary state and hence constrain the progenitors of SNe of different types. We will continue to accumulate high quality SN host photometry so we can improve the sample size and depth for each host and SN type. We will also continue to refine our SED fitting technique and apply this and galaxy evolution diagnostic techniques to the host photometry. Our first efforts will concentrate on the nearby set of SNe Ia that have well determined stretch values [11], but future efforts will explore all types of SNe. 


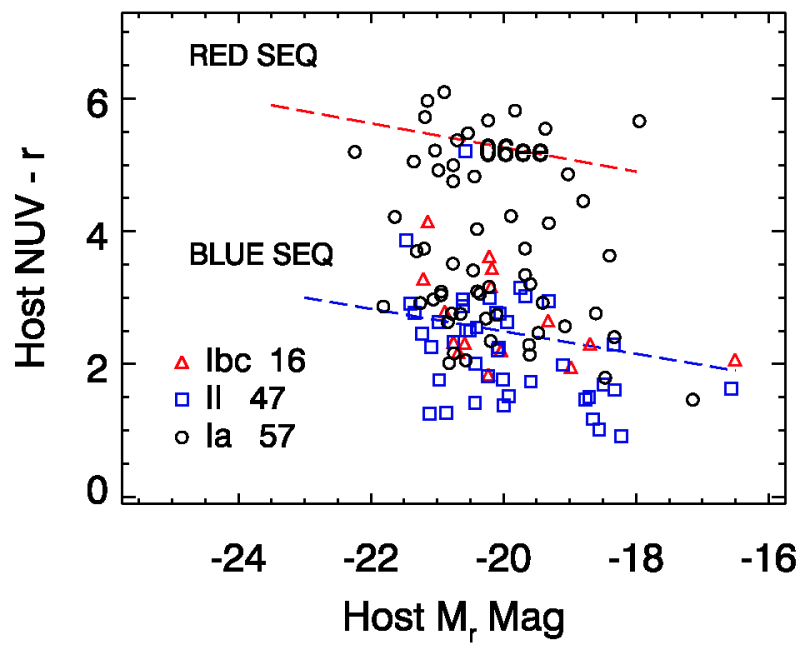

FIGURE 3. SN host NUV-r color versus absolute r-band magnitude $\left(\mathrm{M}_{r}\right)$ for $130 \mathrm{SN}$ hosts coded by type as follows: type Ia - circles, type II - squares, and type Ib/c - triangles. The loci of the red and blue sequences are indicated by the dashed lines.

\section{ACKNOWLEDGMENTS}

We acknowledge the use of the NASA Extragalactic Database and the Infra-Red Science Archive and their vital contribution to this work.

\section{REFERENCES}

1. D. C. Martin, J. Fanson, D. Schiminovich, P. Morrissey, P. G. Friedman, T. A. Barlow, T. Conrow, R. Grange, et al., $A p J 619$, L1 (2005).

2. T. K. Wyder, D. C. Martin, D. Schiminovich, M. Seibert, T. Budavári, M. A. Treyer, T. A. Barlow, K. Forster, et al., ApJS 173, 293 (2007).

3. D. C. Martin, T. Small, D. Schiminovich, T. K. Wyder, P. G. Pérez-González, B. Johnson, C. Wolf, T. A. Barlow, et al., ApJS 173, 415 (2007).

4. A. G. de Paz, S. Boissier, B. F. Madore, M. Seibert, Y. H. Joe, A. Boselli, T. K. Wyder, D. Thilker, et al., ApJS 173, 185 (2007).

5. M. Seibert, in preparation (2009).

6. M. Fioc, and B. Rocca-Volmerange, $A \& A$ 326, 950 (1997).

7. D. L. Borgne, and B. Rocca-Volmerange, $A \& A$ 386, 446 (2002).

8. D. L. Borgne, B. Rocca-Volmerange, P. Prugniel, A. Lançon, M. Fioc, and C. Soubiran, $A \& A$ 425, $881(2004)$

9. A. J. Conley, M. Sullivan, E. Y. Hsiao, J. Guy, P. Astier, D. Balam, C. Balland, S. Basa, et al., ApJ 681, $482(2008)$.

10. D. A. Howell, ApJ 554, L193 (2001).

11. J. D. Neill, in preparation (2009). 